Original article

\title{
Skeletal muscle status, autonomic balance and short-term results of cardiac surgery
}

\author{
Alexey N. Sumin, Pavel A. Oleinik, Andrey V. Bezdenezhnykh \\ Research Institute for Complex Issues of Cardiovascular Diseases, Kemerovo, Russia
}

Received 30 March 2020, Revised 7 July 2020, Accepted 15 July 2020

(C) 2020, Sumin A.N., Oleinik P.A., Bezdenejnykh A.V.

(C) 2020, Russian Open Medical Journal

Abstract: Purpose - To study the association between the status of lower extremities' skeletal muscles and autonomic balance with the short-term results of cardiac surgery patients.

Methods - 64 patients (57 men, median age 63 years) who underwent non-urgent cardiac surgery at the Research Institute for Complex Issues of Cardiovascular Diseases clinic from March 2015 to March 2016 were included in the study. Patients with exacerbation of underlying disease were excluded from the study. Additionally, muscle status of the patients was assessed using static-dynamic tests on a multifunctional training apparatus, and the autonomic nervous system (ANS) state was evaluated using the ORTOexpert program during an active orthostatic test. 9 patients developed complications in the postoperative period; groups with the postoperative complications present and absent were compared according to the studied preoperative indicators.

Results - The following indicators were revealed more often prior to the surgery in the group with the postoperative complications: cardiac arrhythmias $(p=0.023)$, aortic valve regurgitation $(p=0.002)$, left ventricle aneurysm $(p=0.007)$, carotid stenosis $(p=0.036)$, decreased muscle strength of the upper and lower extremities ( $p=0.047$ and $p=0.046)$, sympathetic activation (LF/HF ratio $p=0.028)$, and ANS stress in response to the test.

Conclusion - Low status of skeletal muscles (upper and lower extremities) and ANS sympathetic activation and the overstrain of its adaptive mechanisms were associated with development of postoperative complications after cardiac surgery, along with severity of the underlying cardiac pathology, risk and duration of the surgery.

Keywords: cardiac surgery, postoperative complications, muscle status, autonomic status.

Cite as Sumin AN, Oleinik PA, Bezdenejnykh AV. Skeletal muscle status, autonomic balance and short-term results of cardiac surgery. Russian Open Medical Journal 2020; 9: e0306.

Correspondence to Alexey N. Sumin. Address: 6, Sosnoviy blvd, Kemerovo, 650002, Russia. Phone: +73842643308, +79039408668. E-mail: an_sumin@mail.ru, sumian@kemcardio.ru.

\section{Introduction}

Risk assessment of cardiac surgery is an essential part of the "Heart team" work and affects the choice of optimal surgical tactics and the decision on the possibility of surgery [1]. Current trends in the standards for provision of high-quality, high-tech medical care are such that patients of older age constitute a large, increasing group due to increasing average patient's age [2,3]. Age is considered one of the factors that significantly affect the outcome of the surgery in the existing scales for assessing the surgery risk (EuroSCORE II, STS, etc.) [1]. However, clinicians are well aware of the fact that biological age alone cannot characterize the degree of function loss by the patient; therefore, the term "frailty" was introduced to reflect this parameter [4, 5]. Experience of using "frailty assessment" in a surgical clinic showed that this indicator is able to successfully predict the results of a number of interventions, including heart [6, 7] and aorta [8] surgery, transcatheter aortic valve implantation, and malignant tumor surgery $[9,10]$. Therefore, it seems appropriate to include frailty assessment in the preoperative risk assessment $[2,5]$ and, perhaps, even refine the evaluation criteria in the existing risk assessment scales. However, at present, there are a lot of criteria for frailty and it is not entirely clear which one is more appropriate to use for these purposes. For example, frailty index, Katz index, walking speed, handgrip strength and laboratory tests (plasma albumin and creatinine level) were used for such an assessment [5], and it has been proposed recently to evaluate the crosssectional area of the psoas muscle in multispiral computed tomography [6-8]. Since the muscles of the lower extremities are most important for restoring physical activity after cardiac surgery, assessment of their functional status would be rational, which created the basis for the present study. Another option in assessing the patient's preoperative status is evaluation of their autonomic balance; in particular, heart rate variability indicators have been suggested as a complications prediction tool [11-12]. A comprehensive assessment of muscle and autonomic status in patients with cardiac surgery has not been carried out yet.

Accordingly, the aim of the study was to investigate the association of the lower extremities skeletal muscle state and autonomic status with the short-term results of the operations in the cardiac surgery patients. 


\section{Material and Methods Study design}

The present study included 64 patients ( 57 men and 7 women) aged 38 to 75 years (median age 63 years) who underwent cardiac surgery in the Research Institute for Complex Issues of Cardiovascular Diseases clinic from March 01, 2015 to March 01 2016. The study protocol was approved by the Local Ethics Committee of the Institution and was developed according to the World Medical Association's Declaration of Helsinki on Ethical Principles for Medical Research Involving Human Subjects, 2000 edition. Written informed consent was obtained from all patients prior to them entering the study.

The criteria for inclusion in the study were planned coronary artery bypass grafting or valvular surgery. Patients with age less than 25 and more than 80 years; with exacerbation of underlying disease during the preoperative period (acute myocardial infarction, acute pulmonary embolism, unstable angina, etc.); unable to perform static-dynamic tests due to arthropathies or low pain threshold; with sarcopenia, rhabdomyolysis and other myopathies; with cognitive impairment were excluded from the study.

All patients underwent routine laboratory and instrumental examinations within the framework of inpatient examination standards before surgery, in the early postoperative period and upon discharge from the hospital. Additionally, all subjects before surgery performed a six-minute walk test (6MWT), in accordance with the "American Thoracic Society practical guidelines". The test was carried out indoors, in a direct closed 75 meters long corridor with distance markings. Before the test, patients were instructed. Subjective assessment of the patient's condition while walking was carried out verbally, with standardized phrases every minute, throughout the test. Initial and final control of blood pressure, heart rate, hemoglobin saturation (with digital pulse oximeter), and assessment of the perceived exertion scale were performed.

\section{Static dynamic tests}

Static-dynamic tests were carried out before surgery, on a multifunctional training device, which allows setting the load in the range of 5-100 $\mathrm{kg}$. The measurement was performed in a sitting position, with a tightly fixed pelvis, with knees bent at a $90^{\circ}$ angle. The patient performed knee extensions to the maximum range of motion. The load was increased gradually, in increments of $5 \mathrm{~kg}$, to the maximum tolerated weight. Static tests for flexors and extensors of the lower extremities were carried out after the static-dynamic tests after resting for 5 minutes. Static endurance was measured in seconds holding weights of $50 \%$ of the maximum weight with knees extended until development of severe muscle fatigue.

\section{Autonomic status assessment}

Assessment of autonomic status was carried out using the ORTOexpert software package (NPP "Living Systems"), including a personal computer, and two cardiac sensors (transmitter and pickup sensor) with a radio interface placed on the front surface of the patient's chest.

Before examination the patient was placed in a horizontal position within 5 minutes. Under the control of the rhythmogram, cardiointervals $(\mathrm{Cl})$ were recorded to eliminate radiofrequency interference in the recording. The last two minutes were used to analyze the $\mathrm{Cl}$ rhythmogram. Next, the patient took a vertical position (active orthostatic test - AOT), while continuing to stand motionless for three minutes. Throughout AOT, recording continued until a minimum of $200 \mathrm{Cl}$ was recorded.

During the analysis of the cardiac rhythmogram, the NN intervals (between successive QRS complexes) were estimated. The statistical characteristics of the Cls sequential series (SDNN, RMSSD), as well as the temporal characteristics of heart rate variability (HRV) - Mode (Mo), mode amplitude (AMo), Index of regulation strain (IS) were calculated for both horizontal (resting state) and orthostasis position (load)

Parasympathetic activity of autonomic regulation was assessed by RMSSD. RMSSD is a square root from the sum of squared differences of sequential NN pairs; the higher this indicator, the more active the component of parasympathetic regulation. HRV assessment also was carried out using the following geometric parameters: Mo - the number of the most frequently encountered RR intervals, which makes it possible to assess the state of the patient's regulatory systems; AMo is the fraction of intervals corresponding to the mode value; reflects the stabilizing effect of centralization of heart rhythm control; IS - index of regulatory systems, reflects the degree of the nervous system influence on the heart. IS is quite sensitive to increased tone of the sympathetic nervous system. Physical or emotional stress can increase IS by 50 to $100 \%$, and a significant load elevates it by $5-10$ times. In patients with constant voltage of regulatory systems, resting IS is 400-600 conventional units. In patients with progressive angina pectoris and myocardial infarction, resting IS reaches 1000-1500 units [13].

Parameters of the spectral analysis were estimated in the twominute recording section at rest. The high-frequency (HF) range represents largely the parasympathetic influence on heart rate, while low-frequency (LF) range generally characterizes the sympathetic nervous activity, in particular, the system of vascular tone regulation. The LF/HF ratio is constant and reflects the sympatho-parasympathetic balance, or the index of vagosympathetic interaction. Very slow, low-frequency waves of the 2nd order (VLF - very low frequency), characterize the state of the neurohumoral, slow heart rate regulation system.

Based on the performance of $A O P$, the parameters of the transient process $(\mathrm{KFa}, \mathrm{KFb}, \mathrm{KFc}, \mathrm{KFd})$ and autocorrelation indicators were estimated [13].

The ORTO expert software formed a conclusion about the functional state of the ANS regulatory systems of the body automatically, based on the assessment of vegetative tone, the degree of tension of regulatory systems, the parameters of the transition process, data on the activity of the autonomic nervous system at rest and during AOT.

\section{Patients' basic characteristics}

Presence of one or more perioperative complications was determined a criterion for sorting patients into groups, i.e. the combined endpoint (CEP) included development of stroke, myocardial infarction (MI), persistent arrhythmias and heart block, development of multiple organ dysfunction syndrome (MODS), death. Thus, 2 groups were formed - with CEP $(n=9)$ and without CEP ( $n=55)$. The studied groups were compared according to the main anthropometric and demographic indicators, presence of risk factors for atherosclerosis, concomitant pathology, history of 
atherothrombotic events, laboratory and instrumental examination data and the immediate results of cardiac surgery.

No difference was found in gender-age characteristics of the two groups (Table 1). The groups were comparable in main clinical indicators as well; history of myocardial infarction was detected slightly more often in the group without CEP, but the differences were not statistically significant $(p=0.067)$. In the same time, during the preoperative examination, arrhythmias were more often detected in the group with presence of CEP $(p=0.023)$. The groups did not differ in prevalence of severe angina pectoris, percutaneous coronary interventions ( $\mathrm{PCl}$ ) and history of stroke, atrial fibrillation and diabetes.

While performing laboratory tests (Table 2), no significant differences between the groups were noted as well. According to instrumental examinations (Table 3 ), in the CEP group during the initial examination, severe aortic valve insufficiency $(p=0.002)$ and aneurysm of the left ventricle $(p=0.007)$ were significantly more often detected. Manifestations of peripheral atherosclerosis, such as hemodynamically insignificant ICA stenosis, were significantly more often observed in the group with CEP $(p=0.036)$. At the same time, the groups did not differ in prevalence of the lower extremities arteries stenosis.

\section{Statistical analysis}

All statistical analyses were calculated with STATISTICA 10.0 (Dell Software, Inc., Round Rock, TX, USA). The Shapiro-Wilk test was used to test data for normal distribution. Since the distribution for all quantitative variables differed from normal, they are presented as median, lower and upper quartile - Me (LQ UQ). Categorical data were reported as percentages. Differences in quantitative data between NMES groups and controls were evaluated using the Mann-Whitney test. Nominal and binary signs were compared using the $\chi 2$ criterion with Yates correction for small samples. The relationship of possible factors with likelihood of developing a combined endpoint was evaluated in a logistic regression model. The suggested predictors are: gender, age, $\mathrm{LF} / \mathrm{HF}$, indicators of muscle status, Amo at rest and in orthostasis, IS at rest and in orthostasis, cardiopulmonary bypass duration and glomerular filtration rate. The preliminary identification of possible correlations between the proposed predictors was carried out, then the regression models were formed taking into account the revealed correlations. Multivariate analysis included variables for which the criterion of statistical significance in univariate analysis was less than 0.05

Table 1. Baseline characteristics of patients

\begin{tabular}{|c|c|c|c|}
\hline & Group 1 (with CEP) $(n=9)$ & Group 2 (without CEP) $(n=55)$ & P-level \\
\hline Male & $7(77.78)$ & $50(90.91)$ & 0.242 \\
\hline Female & $2(22.22)$ & 5 (9.09) & 0.242 \\
\hline Age, years & $64.0(60.0,65.0)$ & $61.0(57.0,65.0)$ & 0.290 \\
\hline Body mass index, $\mathrm{kg} / \mathrm{m}^{2}$ & $25.67(21.68,27.44)$ & $27.61(24.90,30.86)$ & 0.135 \\
\hline $\mathrm{FC}$ angina pectoris $\geq 3$ & $1(11.11)$ & $10(18.18)$ & 0.784 \\
\hline Old myocardial infarction & $4(44.44)$ & $41(74.54)$ & 0.067 \\
\hline Creatinine, $\mu \mathrm{mol} / \mathrm{L}$ & $85.0(75.0,90.0)$ & $78.0(71.0,86.0)$ & 0.579 \\
\hline $\mathrm{GFR}, \mathrm{ml} / \mathrm{min} / 1.73 \mathrm{~m}^{2}$ & $80.78(73.95,90.43)$ & $87.48(78.04,97.65)$ & 0.496 \\
\hline AG duration $\geq 5$ yeas & $4(44.44)$ & $24(43.64)$ & 0.910 \\
\hline $\mathrm{PCl}$ history & $3(33.33)$ & $14(25.46)$ & 0.620 \\
\hline Stroke history & $1(11.11)$ & $4(7.27)$ & 0.691 \\
\hline $\mathrm{CHF} \geq 2 \mathrm{a}$ & $7(77.78)$ & $33(60)$ & 0.307 \\
\hline Rhythm disturbances & $5(55.56)$ & $11(20)$ & 0.023 \\
\hline Atrial fibrillation & $0(0)$ & $3(5.45)$ & 0.473 \\
\hline Diabetes mellitus & $1(11.11)$ & $9(16.36)$ & 0.687 \\
\hline Peripheral arterial disease & $1(11.11)$ & $5(9.09)$ & 0.847 \\
\hline \multicolumn{4}{|c|}{ Operative procedure } \\
\hline CABG & $5(55.56)$ & $35(63.64)$ & 0.447 \\
\hline CABG + valve replacement & $2(22.22)$ & $3(5.45)$ & 0.074 \\
\hline CABG + radiofrequency ablation & $0(0)$ & $4(7.27)$ & 0.412 \\
\hline Multivalve operations & $1(11.11)$ & $3(5.45)$ & 0.494 \\
\hline Aortic valve replacement & $0(0)$ & $2(3.64)$ & 0.568 \\
\hline Mitral valve replacement & $0(0)$ & $2(3.64)$ & 0.568 \\
\hline
\end{tabular}

Quantitative data are presented as median with lower and upper quartiles - Me (LQ, UQ), binary variables - as frequencies in absolute values and in percentage $-\mathrm{n}(\%)$. CEP, combined endpoint; $\mathrm{FC}$, functional class, GFR, glomerular filtration rates; $\mathrm{AG}$, arterial hypertension; $\mathrm{CHF}$, chronic heart failure; PCl, percutaneous coronary intervention; CABG, coronary artery bypass grafting.

Table 2. Baseline characteristics of patients (laboratory tests)

\begin{tabular}{|c|c|c|c|}
\hline & Group 1 (with CEP) $(n=9)$ & Group 2 (without CEP) $(n=55)$ & P-level \\
\hline Glucose (mmol/L) & $5.65(5.35,6.50)$ & $5.95(5.10,6.50)$ & 0.681 \\
\hline Urea (mmol/L) & $6.3(5.4,7.8)$ & $6.5(5.2,7.4)$ & 0.856 \\
\hline Creatinine $(\mu \mathrm{mol} / \mathrm{L})$ & $85.0(75.0,90.0)$ & $78.0(71.0,86.0)$ & 0.579 \\
\hline Total bilirubin $(\mu \mathrm{mol} / \mathrm{L})$ & $16.2(9.2,21.8)$ & $14.4(10.7,18.7)$ & 0.946 \\
\hline Direct bilirubin $(\mu \mathrm{mol} / \mathrm{L})$ & $5.05(4.35,6.15)$ & $4.40(3.70,6.50)$ & 0.836 \\
\hline Aspartate Aminotransferase (U/L) & $21.0(18.5,24.0)$ & $22.0(19.0,29.0)$ & 0.672 \\
\hline Alanine Aminotransferase (U/L) & $27.5(17.0,37.5)$ & $26.0(20.0,37.0)$ & 0.619 \\
\hline Cholesterol (mmol/L) & $4.3(3.8,4.8)$ & $4.4(3.6,5.2)$ & 0.623 \\
\hline High density lipoproteins (mmol/L) & $1.37(0.78,1.91)$ & $0.97(0.78,1.27)$ & 0.203 \\
\hline Low density lipoproteins (mmol/L) & $2.25(1.36,2.33)$ & $2.53(1.98,3.15)$ & 0.138 \\
\hline Triglycerides (mmol/L) & $1.34(0.79,1.39)$ & $1.52(1.11,1.84)$ & 0.143 \\
\hline Erythrocyte sedimentation rate $(\mathrm{mm} / \mathrm{h})$ & $4.0(4.0,10.0)$ & $7.0(4.0,10.0)$ & 0.741 \\
\hline
\end{tabular}

Data are presented as median with lower and upper quartiles - Me (LQ, UQ). CEP, combined endpoint. 
Table 3 Baseline characteristics of patients (echocardiography and Doppler ultrasound of blood vessels)

\begin{tabular}{|c|c|c|c|}
\hline & Group 1 (with CEP) (n=9) & Group 2 (without CEP) ( $n=55)$ & P-level \\
\hline Left Atrial Diameter, $\mathrm{cm}$ & $4.6(3.90,4.70)$ & $4.3(4.0,4.70)$ & 0.882 \\
\hline Left Ventricle End-Diastolic Volume, ml & $209.0(160.0,247.0)$ & $180.0(141.0,201.0)$ & 0.371 \\
\hline Left Ventricle End-Systolic Volume, ml & $102.0(88.0,113.0)$ & $76.5(51.0,132.50)$ & 0.531 \\
\hline Interventricular Septal Thickness, mm & $1.2(1.0,1.25)$ & $1.2(1.10,1.20)$ & 0.520 \\
\hline Posterior wall thickness, $\mathrm{mm}$ & $1.2(1.0,1.25)$ & $1.2(1.0,1.20)$ & 0.394 \\
\hline Right ventricle mid-diameter, $\mathrm{mm}$ & $1.8(1.80,1.90)$ & $1.85(1.80,2.0)$ & 0.564 \\
\hline Aorta, cm & $3.6(3.20,3.80)$ & $3.6(3.50,3.80)$ & 0.545 \\
\hline Left ventricular ejection fraction, $\%$ & $56.0(50.0,63.0)$ & $58.0(50.0,64.0)$ & 0.621 \\
\hline Pulmonary artery systolic pressure, $\mathrm{mmHg}$ & $25.0(22.0,28.0)$ & $28.0(24.0,34.0)$ & 0.488 \\
\hline Mitral regurgitation $\geq$ grade 2 & $3(33.33)$ & $9(16.36)$ & 0.227 \\
\hline Aortic valve regurgitation $\geq$ grade 2 & $3(33.33)$ & $2(3.64)$ & 0.002 \\
\hline Left ventricular aneurysm & $2(22.22)$ & $1(1.82)$ & 0.007 \\
\hline Left ventricular myocardial mass, $g$ & $293.8(273.3,349.0)$ & $323.8(256.0,369.0)$ & 0.455 \\
\hline Internal carotid artery stenosis $\geq 30 \%$ & $6(66.67)$ & $19(34.55)$ & 0.036 \\
\hline Stenosis of lower limb arteries & $2(22.22)$ & $13(23.64)$ & 0.656 \\
\hline
\end{tabular}

Quantitative data in Tables 3, 4, 5, and 6 are presented as median with lower and upper quartiles - Me (LQ, UQ), binary variables - as frequencies in absolute values and in percentage $-\mathrm{n}(\%)$. CEP, combined endpoint.

Table 4. Muscle strength of the lower and upper extremities and 6MWT results before surgery

\begin{tabular}{|c|c|c|c|}
\hline & Group 1 (with CEP) (n=9) & Group 2 (without CEP) (n=55) & $p$ value \\
\hline Distance of 6MWT, m & $320.0(290.0,355.0)$ & $320.0(300.0,390.0)$ & 0.690 \\
\hline Distance of $6 \mathrm{MWT} \geq 300 \mathrm{~m}$ & $6(66.67)$ & $42(76.34)$ & 0.785 \\
\hline Handgrip strength, kg & $20.0(15.0,25.0)$ & $25.0(20.0,30.0)$ & 0.047 \\
\hline Knee extensors strength, kg & $20.0(15.0,25.0)$ & $25.0(20.0,30.0)$ & 0.046 \\
\hline Knee extensors endurance, sec & $63.0(38.0,113.0)$ & $55.0(40.0,86.0)$ & 0.690 \\
\hline Knee extensors work, kg*sec & $950.0(610.0,2025.0)$ & $1375.0(810.0,2370.0)$ & 0.470 \\
\hline
\end{tabular}

CEP, combined endpoint, 6MWT, 6-minute walk test.

Table 5. Autonomic status indicators before surgery in groups with and without CEP

\begin{tabular}{|c|c|c|c|}
\hline & Group 1 (with CEP) (n=9) & Group 2 (without CEP) $(n=55)$ & P-level \\
\hline $\mathrm{TF}, \mathrm{ms}^{2}$ & $1955.0(1087.0,4137.0)$ & $1158.5(566.0,2144.0)$ & 0.779 \\
\hline $\mathrm{VLF}, \mathrm{ms}^{2}$ & $1275.0(946.0,2072.0)$ & $693.5(258.0,1571.0)$ & 0.901 \\
\hline $\mathrm{LF}, \mathrm{ms}^{2}$ & $504.0(259.0,1859.0)$ & $248.5(130.0,455.0)$ & 0.726 \\
\hline $\mathrm{LF} / \mathrm{HF}$ & $7.25(3.85,8.34)$ & $2.71(1.63,5.33)$ & 0.028 \\
\hline$L F, \%$ & $88.0(79.0,89.0)$ & $73.0(62.0,84.0)$ & 0.013 \\
\hline$H F, \%$ & $12.0(11.0,21.0)$ & $27.0(16.0,38.0)$ & 0.013 \\
\hline $\mathrm{KFb}$ & $-29.0(-94.0,18.0)$ & $1.5(0.0,47.0)$ & 0.162 \\
\hline KFc & $0.0(-1.0,8.0)$ & $3.0(0.0,10.0)$ & 0.818 \\
\hline KFd & $0.0(-13.0,7.0)$ & $-11.0(-56.0,0.0)$ & 0.761 \\
\hline Amo supine, $\%$ & $52.0(44.0,61.0)$ & $68,0(50.0,84.0)$ & 0.026 \\
\hline IS supine & $150.9(95.0,61.0)$ & $265.85(118.8,488.0)$ & 0.094 \\
\hline Amo upright, $\%$ & $60.0(55.0,72.0)$ & $60.5(49.0,84.0)$ & 0.593 \\
\hline IS upright & $393.0(155.50,523.80)$ & $258.45(138.20,632.40)$ & 0.390 \\
\hline Amo supine-upright, \% & $-15.0(-20.0,-9.0)$ & $-1.5(-13.0,13.0)$ & 0.145 \\
\hline
\end{tabular}

CEP, combined endpoint; TF, Total Frequency; VLF, Very Low Frequency; LF, Low Frequency; HF, High Frequency; Kfa, first phase of the calculated transient parameter coefficients during AOT; Kfb, second phase of the calculated transient parameter coefficients during AOT; Kfc, third phase of the calculated transient parameter coefficients during AOT; Kfd, fourth phase of the calculated transient parameter coefficients during AOT; Amo, mode amplitude; IS, Index of regulation strain.

Table 6. Perioperative characteristics of surgery in groups with and without CEP

\begin{tabular}{|c|c|c|c|}
\hline & Group 1 (with CEP) $(n=9)$ & Group 2 (without CEP) $(n=55)$ & P-level \\
\hline EuroSCORE, \% & $2.32(2.57,5.46)$ & $1.89(1.33,2.37)$ & 0.005 \\
\hline EuroSCORE, units & $4.5(4.0,5.50)$ & $3.0(2.0,4.0)$ & 0.005 \\
\hline Cardiopulmonary bypass duration, min & $128.0(86.0,185.0)$ & $81.0(61.0,98.0)$ & 0.002 \\
\hline Aortic cross-clamp time, min & $104.0(49.0,147.0)$ & $53,0(38.0,68.0)$ & 0.007 \\
\hline Congestive heart failure & $4(44.44)$ & $0(0)$ & $<0.001$ \\
\hline Atrial fibrillation & $2(22.22)$ & $0(0)$ & $<0.001$ \\
\hline Atrial flutter & $1(3.70)$ & $0(0)$ & 0.013 \\
\hline MODS & $1(3.70)$ & $0(0)$ & 0.013 \\
\hline Hydrothorax & $4(44.44)$ & $31(56.36)$ & 0.505 \\
\hline Pneumonia & $2(22.22)$ & $0(0)$ & $<0.001$ \\
\hline Stroke & $1(3.70)$ & $0(0)$ & 0.013 \\
\hline Death & $1(3.70)$ & $0(0)$ & 0.013 \\
\hline
\end{tabular}

CEP, combined endpoint; MODS, multiple organ dysfunction syndrome. 
Table 7. Associations between basal characteristics and risk of CEP development

\begin{tabular}{|c|c|c|}
\hline Characteristics & $\begin{array}{c}\text { Odds ratio } \\
(-95 \% \mathrm{Cl} ;+95 \% \mathrm{Cl})\end{array}$ & $p$ \\
\hline \multicolumn{3}{|c|}{ Univariate logistic regression analysis } \\
\hline Old myocardial infarction & $0.035(0.082 ; 1.502)$ & 0.151 \\
\hline Rhythm disturbances & $1.769(0.377 ; 8.292)$ & 0.461 \\
\hline Aortic valve regurgitation $\geq$ grade 2 & $1.687(0.160 ; 18.828)$ & 0.658 \\
\hline Left ventricular aneurysm & $3.563(0.276 ; 46.029)$ & 0.321 \\
\hline Internal carotid artery stenosis $\geq 30 \%$ & $9.545(1.030 ; 88.467)$ & 0.043 \\
\hline Handgrip strength & $0.893(0.786 ; 1.001)$ & 0.048 \\
\hline Knee extensors strength & 1.012 (0.997; 1.029) & 0.098 \\
\hline $\mathrm{LF} / \mathrm{HF}$ & $1.104(0.944 ; 1.292)$ & 0.207 \\
\hline $\mathrm{HF}, \%$ & $0.999(0.997 ; 1.000)$ & 0.628 \\
\hline Amo supine & $0.958(0.920 ; 0.998)$ & 0.037 \\
\hline IS supine & 0.996 (0.990; 1.000) & 0.096 \\
\hline Amo upright & $0.991(0.957 ; 1.025)$ & 0.587 \\
\hline IS upright & $0.999(0.997 ; 1.000)$ & 0.398 \\
\hline EuroSCORE, \% & $1.249(0.834 ; 1.868)$ & 0.270 \\
\hline EuroSCORE, units & $1.643(1.003 ; 2.694)$ & 0.043 \\
\hline Cardiopulmonary bypass duration & $1.010(1.001 ; 1.022)$ & 0.068 \\
\hline Aortic cross-clamp time & $1.009(0.998 ; 1.023)$ & 0.138 \\
\hline CABG + valve replacement & $5.142(0.701 ; 37.713)$ & 0.112 \\
\hline \multicolumn{3}{|c|}{ Multivariable logistic models; $p=0.001$} \\
\hline LF/HF & $1.175[0.972 ; 1.420]$ & 0.088 \\
\hline Amo supine & $0.950[0.905 ; 0.997]$ & 0.034 \\
\hline Handgrip strength & $0.863[0.750 ; 0.993]$ & 0.036 \\
\hline
\end{tabular}

CEP, combined endpoint; $\mathrm{Cl}$, confidence interval; $\mathrm{HF}$, High Frequency; $\mathrm{LF}$, Low Frequency; Amo, mode amplitude; IS, Index of regulation strain; CABG, coronary artery bypass grafting.

\section{Results}

The physical tests found no difference in endurance of the lower extremities skeletal muscles or the distance covered by the results of 6MWT between the groups. At the same time, the group with CEP was characterized by lower strength of the skeletal muscles of the upper and lower extremities $(p=0.047$ and $p=0.046$, respectively; Table 4).

When assessing the vegetative status (Table 5) at the preoperative stage, an initially more pronounced sympathetic activation can be noted in the CEP group based on the percentage of the low and high frequency ranges ( 88 to $12 \%$ in the CEP group, 73 to $27 \%$ in the no CEP group, $p=0.013$ ), which reflects the degree of the sympathetic component activity in the regulation of the autonomic nervous system (ANS). Also, larger values of the total power of the HRV frequency spectrum (TF) in the CEP group were revealed as compared to the non-CEP group, although these differences were not statistically significant (1955.0 and 1158.5, $\mathrm{p}=0.779$ ).

It is also worth paying attention to the mode amplitude parameter (Amo), which illustrates distribution of the most frequently recorded cardiointervals (CI), and the stress index (IS) calculated on its basis for characterizing the stress adaptation of regulatory systems. Initially, at rest, IS was lower in the CEP group (150.9 and 265.85, $p=0.094$ ), but after transitioning to the orthostatic position it increased more than twice and surpassed the number in the group without CEP (393.0 and 258.45, $p=0.390$ ). Values of the transition process parameters also had no significantly significant differences between groups ( $\mathrm{Kfa} p=0.628$; KFb $p=0.162 ; \mathrm{KFc} p=0.818 ; \mathrm{KFd} p=0.761$ ).

The risk of surgery was initially higher in the CEP group when assessed according to the EuroSCORE II scale $(p=0.005)$, so, expectedly, the cardiopulmonary bypass duration and aortic clamping time were longer ( $p=0.002$ and $p=0.007$, respectively). The spectrum of complications is presented in Table 6; their presence is explained primarily by the severity of the underlying disease and the duration of the operation.

According to the data obtained with the univariate logistic regression analysis, the most likely factors associated with the development of CEP in this study are poor functional state of skeletal muscles (particularly upper limbs), internal carotid artery stenosis stenosis of 30 percent or more; higher EuroSCORE surgery risk, cardiopulmonary bypass duration and HRV indices characterizing the overstrain of the mechanisms of regulation of the ANS and the tendency to sympathicotonia (Table 7).

Given the strong correlation between the strength indicators of the upper and lower extremities muscles, only the upper extremities strength, that demonstrated significance in the univariate analysis, was included in the multivariate model. Multivariate regression analysis allowed us to establish the relationship of poor skeletal muscle status, pronounced sympathicotonia, overstrain of adaptogenic ANS mechanisms and a high incidence of significant, life-threatening postoperative complications (Table 7).

\section{Discussion}

Our study shows that, in addition to generally accepted indicators (risk and duration of surgery), such parameters as muscle strength of the upper and lower extremities and autonomic imbalance are associated with immediate results of cardiac surgery. It is not much of a surprise, if we consider the reduced functional state of these muscle groups as one of the manifestations of patients' fragility. It was shown in a study by Foldyna et al. that patients with the smallest cross-sectional area of the psoas muscle according to computed tomography had the highest mortality within 1-2 years after transcatheter aortic valve implantation [14]. In a mixed group of patients (open-heart aortic valve surgery and transcatheter implantation of the aortic valve), sarcopenia was also evaluated by the total psoas area during computed tomography. Independent predictors of a 30-day outcome (death, stroke, acute renal failure, prolonged mechanical ventilation, deep wound infection) were STS scale risk (OR 91.1; $p=0.02$ ) and total psoas area (OR 0.5; $p=0.024)$. The two-year survival rate was $85.7 \%$ in patients with sarcopenia compared to $93.8 \%$ in patients without sarcopenia $(p=0.02)$; accordingly, the total psoas area was an independent predictor of long-term survival (OR 0.47; $p=0.02$ ) [15]. During aortic valve replacement surgery, patients with sarcopenia (psoas index values within the lower quartile) had a large annual mortality rate $(31.9 \%$ versus $16.9 \% \mathrm{p}=0.03)$, with the psoas index having an independent prognostic effect on the annual (OR $0.84 ; p=0.02$ ) and long-term (OR 0.92; $p=0.04$ ) mortality [7]. As we can see, all these methods are able to predict the risk of complications. According to our data, knee extensor muscle strength can also be used to assess the likelihood of developing complications after cardiac surgery and, in our opinion, may be preferable in this group of patients. There are studies that have shown that the electric skeletal muscle stimulation (EMS) in patients after cardiac surgery are safe [16] and can improve skeletal muscle metabolism [17]. Therefore, assessing the muscles' status before and after EMS allows to evaluate its effectiveness. This will help to answer another clinically important question - is it possible to improve the results of surgical treatment by preoperative training of skeletal muscles? The data available so far is mainly focused on the postoperative period and refers to patients with complicated postoperative 
period, while the use of EMS in rehabilitation is extremely limited and refers to non-cardiac surgery [18].

Data on the possibility of using HRV indicators in predicting the outcome of cardiac surgery is limited, despite a fairly large number of studies evaluating HRV in these patients. The fact is that they mainly investigate the peri- and postoperative period. It was previously shown that cardiac surgery itself leads to a decrease in HRV indices, which are later restored to the initial level [19]. Failure of the HRV indices to recover may be an adverse surgery outcome factor [19] (although this opinion is debated [20]). Preoperative HRV scores have been shown to be significant in a study by de Godoy MF et al. [21], who showed that nonlinear domains of HRV indices can help to identify a group of patients with a high risk of perioperative complications of CABG.

In our study, when directly comparing the prognostic significance of muscle status indicators and HRV data, the possibility of using these indicators in combination is convincingly shown. It should be noted that the great prognostic significance of HRV indicators was noted. Perhaps this should not be surprising, since sympathetic activation can develop not only with a decrease in the pumping function of the heart, but also with skeletal muscle dysfunction [22]. Therefore, autonomic dysfunction can be expected to be more pronounced (due to a combination of central and peripheral influences) than the degree of muscle maladaptation. Ultimately, when assessing the parameters of the transition process, both groups go through the stage of adaptive activation of the ANS, but for the group with initial pronounced sympathicotonia, activation occurs with a high tension of regulatory systems, with a slower, probably not clinically significant, speed of fast adaptive reactions. Given the dynamic changes in the mode amplitude indicators (Amo) and IS, we can talk about decreased adaptive ability of the ANS to adequately respond to loads of any type in the group having complications after surgery. So the role of the ANS regulation maladaptation in the formation of early postoperative complications requires further study.

Study Limitations. The main limitations of the study are the short duration of observation and the small sample size of patients, which to some extent affects the prognostic value of the results obtained on the dynamics of muscle and autonomic parameters. Only 64 patients who underwent open coronary revascularization or correction of valvular heart disease were included in this study. The presented comparison groups are rather small; therefore, the use of multiple regression analysis to study the dynamics of indicators of muscle and vegetative status is complicated. The groups included in the study are quite heterogeneous, which suggests the possible impact of surgical procedures on the contribution of muscle status and autonomic balance to immediate results. However, the study of Shvartz V.A. et al. [12] with assessment of the pre and postoperative cardiovascular autonomic parameters showed no differences in the groups of patients undergoing CABG and valve prosthetics. Autonomic balance indices did not depend on the type and volume of surgical intervention and clinical characteristics of the patients. A more accurate study of this issue will be necessary in more homogeneous groups of patients in subsequent studies. Also, another limitation is the lack of studying the daily variability of autonomic indicators and the use of only short data on HRV during AOT.

\section{Conclusion}

Perioperative complications (development of acute cerebrovascular accident, myocardial infarction, persistent arrhythmias and conduction disorders, multiple organ failure syndrome, death) occurred in 9 out of 64 cases after cardiac surgery. Not only the severity of the underlying cardiac pathology, the risk and duration of the operation, but also muscle strength of the upper and lower extremities, as well as sympathetic activation of the autonomic nervous system and its adaptive reserve were associated with development of CEP. The possibility of improving the postoperative outcomes by improving the functional status of skeletal muscles in the preoperative period, and the effect of muscle status on the autonomic balance requires further analysis.

\section{Conflict of interest}

All authors declare no conflict of interest.

\section{References}

1. Neumann FJ, Sousa-Uva M, Ahlsson A, Alfonso F, Banning AP, Benedetto U, et al.; ESC Scientific Document Group. 2018 ESC/EACTS Guidelines on myocardial revascularization. Eur Heart J 2019; 40(2): 87-165. https://doi.org/10.1093/eurheartj/ehy394.

2. Cockburn J, Hildick-Smith D, Trivedi U, de Belder A. Coronary revascularisation in the elderly. Heart 2017; 103(4): 316-324. https://doi.org/10.1136/heartjnl-2015-308999.

3. Gajfulin RA, Sumin AN, Ivanov SV, Barbarash LS. Survival after surgical treatment of multifocal atherosclerosis in different age groups. Complex Issues of Cardiovascular Diseases 2017; 6(2): 6-18. Russian. https://www.elibrary.ru/item.asp?id=29422229.

4. Clegg A, Young J, Iliffe S, Rikkert MO, Rockwood K. Frailty in elderly people. Lancet 2013; 381(9868): $752-762$ https://doi.org/10.1016/s0140-6736(12)62167-9.

5. Furukawa $\mathrm{H}$, Tanemoto $\mathrm{K}$. Frailty in cardiothoracic surgery: systematic review of the literature. Gen Thorac Cardiovasc Surg 2015; 63(8): 425 433. https://doi.org/10.1007/s11748-015-0553-8.

6. Zuckerman J, Ades M, Mullie L, Trnkus A, Morin JF, Langlois $\mathrm{Y}$, et al. Psoas Muscle Area and Length of Stay in Older Adults Undergoing Cardiac Operations. Ann Thorac Surg 2017; 103(5): 1498-1504. https://doi.org/10.1016/j.athoracsur.2016.09.005.

7. Hawkins RB, Mehaffey JH, Charles EJ, Kern JA, Lim DS, Teman NR, et al. Psoas Muscle Size Predicts Risk-Adjusted Outcomes After Surgical Aortic Valve Replacement. Ann Thorac Surg 2018; 106(1): 39-45. https://doi.org/10.1016/j.athoracsur.2018.02.010.

8. Drudi LM, Phung K, Ades M, Zuckerman J, Mullie L, Steinmetz OK, et al. Psoas Muscle Area Predicts All-Cause Mortality After Endovascular and Open Aortic Aneurysm Repair. Eur J Vasc Endovasc Surg 2016; 52(6): 764-769. https://doi.org/10.1016/j.ejvs.2016.09.011.

9. Jones KI, Doleman B, Scott S, Lund JN, Williams JP. Simple psoas crosssectional area measurement is a quick and easy method to assess sarcopenia and predicts major surgical complications. Colorectal Dis 2015; 17(1): O20-O26. https://doi.org/10.1111/codi.12805.

10. Amini N, Spolverato G, Gupta R, Margonis GA, Kim Y, Wagner D, et al. Impact Total Psoas Volume on Short- and Long-Term Outcomes in Patients Undergoing Curative Resection for Pancreatic Adenocarcinoma: a New Tool to Assess Sarcopenia. J Gastrointest Surg 2015; 19(9): 1593-1602. https://doi.org/10.1007/s11605-015-2835-y.

11. Nenna A, Lusini M, Spadaccio C, Nappi F, Greco SM, Barbato R, et al. Heart rate variability: a new tool to predict complications in adult cardiac surgery. J Geriatr Cardiol 2017; 14(11): 662-668. https://doi.org/10.11909/j.issn.1671-5411.2017.11.005.

12. Shvartz VA, Kiselev AR, Karavaev AS, Vulf KA, Borovkova EI, Prokhorov $M D$, et al. Comparative study of short-term cardiovascular autonomic control in cardiac surgery patients who underwent coronary artery 
bypass grafting or correction of valvular heart disease. J Cardiovasc Thorac Res 2018; 10(1): 28-35. https://doi.org/10.15171/jcvtr.2018.05.

13. Bayevsky RN, Ivanov GG. Cardiac rhythm variability: the theoretical aspects and the opportunities of clinical application (lecture). Ultrasound and Functional Diagnostics 2001; (3): 106-127. https://www.elibrary.ru/item.asp?id=25990135.

14. Foldyna B, Troschel FM, Addison D, Fintelmann FJ, Elmariah S, Furman $D$, et al. Computed tomography-based fat and muscle characteristics are associated with mortality after transcatheter aortic valve replacement. J Cardiovasc Comput Tomogr 2018; 12(3): 223-228. https://doi.org/10.1016/j.jcct.2018.03.007.

15. Paknikar R, Friedman J, Cron D, Deeb GM, Chetcuti S, Grossman PM, et al. Psoas muscle size as a frailty measure for open and transcatheter aortic valve replacement. J Thorac Cardiovasc Surg 2016; 151(3): 745751. https://doi.org/10.1016/i.jtcvs.2015.11.022.

16. Schardong J, Kuinchtner GC, Sbruzzi G, Plentz RDM, Silva AMVD. Functional electrical stimulation improves muscle strength and endurance in patients after cardiac surgery: a randomized controlled trial. Braz J Phys Ther 2017; 21(4): 268-273. https://doi.org/10.1016/j.bjpt.2017.05.004.

17. Fontes Cerqueira TC, Cerqueira Neto ML, Cacau LAP, Oliveira GU, Silva Júnior WMD, Carvalho Vo, et al. Ambulation capacity and functional outcome in patients undergoing neuromuscular electrical stimulation after cardiac valve surgery: A randomised clinical trial. Medicine (Baltimore) 2018; $\quad$ 97(46): https://doi.org/10.1097/md.0000000000013012.

18. Desmeules F, Hall J, Woodhouse LJ. Prehabilitation improves physical function of individuals with severe disability from hip or knee osteoarthritis. Physiother Can 2013; 65(2): 116-124. https://doi.org/10.3138/ptc.2011-60.

19. Lakusic N, Mahovic D, Kruzliak P, Cerkez Habek J, Novak M, Cerovec D. Changes in Heart Rate Variability after Coronary Artery Bypass Grafting and Clinical Importance of These Findings. Biomed Res Int 2015; 2015: 680515. https://doi.org/10.1155/2015/680515.

20. Milicevic G, Fort L, Majsec M, Bakula V. Heart rate variability decreased by coronary artery surgery has no prognostic value. Eur $J$ Cardiovasc Prev Rehabil 2004; 11(3): 228-232. https://doi.org/10.1097/01.hjr.0000129743.53464.c0.

21. de Godoy MF, Takakura IT, Correa PR, Machado MN, Miranda RC, Brandi AC. Preoperative nonlinear behavior in heart rate variability predicts morbidity and mortality after coronary artery bypass graft surgery. Med Sci Monit 2009; 15(3): CR117- CR122. https://pubmed.ncbi.nlm.nih.gov/19247242.

22. Notarius CF, Millar PJ, Floras JS. Muscle sympathetic activity in resting and exercising humans with and without heart failure. Appl Physiol Nutr Metab 2015; 40(11): 1107-1115. https://doi.org/10.1139/apnm2015-0289.

Authors:

Alexey N. Sumin - MD, DSc, Head of the Multifocal Atherosclerosis Department, Research Institute for Complex Issues of Cardiovascular Diseases, Kemerovo, Russia. https://orcid.org/0000-0002-0963-4793.

Pavel A. Oleinik - MD, Phd student, Research Institute for Complex Issues of Cardiovascular Diseases, Kemerovo, Russia. https://orcid.org/00000002-8196-5570.

Andrey V. Bezdenezhnykh - MD, PhD, Senior Researcher, Research Institute for Complex Issues of Cardiovascular Diseases, Kemerovo, Russia. https://orcid.org/0000-0002-4420-4350. 